\title{
Some remarks on the motions of the stars in the Perseus group
}

\author{
by E. A. KREIKEN \\ (Department of Astronomy)
}

\begin{abstract}
In the present paper an attempt was made to determine the coeffieient of space absorption in the direction of the moving Perseus group. The opinion of Smart is confirmed that the group is not a moving cluster. For $\alpha$ a value of $a=0,00103 \mathrm{mag} / \mathrm{parsec}$ is obtained. The absolute magnitudes resulting from the method applied in this paper seem to be overestimated. The main uncertainty in the values of the absorption coefficient and of the absolute magnitudes is due to imperfect knowledge of the mean space velocity of the stars under consideration. The main conclusion is that the method for the determination of $a$ as developed in a previous paper promised to lead to good results.

$$
*^{*} *
$$

Ozet: Bu makalede, hareket eden Perseus grubu istikametinde, uzay absorpsiyon katsayısıı tayin etmeğe teşebbüs edilmiştir. Smart'ın grubun hareket eden bir grup olmadığı hakkındaki fikri teyid edilir. $a$ için $a=0,00103$ mag/parsek değeri elde edilir. Bu makalede tatbik edilen metoddan çikan mutlak kadirler fazla tahmin edilmiş olarak görülürler. Absorpsiyon katsayısı vo mutlak kadir degerlerindeki bał̧lıca belirsizlik tetkik edilen yıldızlar için ortalama uzay hizı hakkındaki bilgimizin mükemmel olmamasından ileri gelir Elde edilen esas netice; a nı tayiai için evvelki makalede geliştirilen metodan iyi neticeler verecę̆ini göstermesidir.
\end{abstract}

\section{§ 1. Introduction}

The parallaxes derived from the proper motions of members of moving clusters are among the most reliable we have at our disposal. Apart from the moving clusters for which a common space velocity has been firmly established, there are several groups of stars which to a certain extent seem to have a com. mon space velocity, but for which it is doubtful whether they can be considered as a moving cluster. In such cases we may 
have to deal with a group of stars chosen from a wide. field on the basis of their apparently nearly parallel proper motions. In such cases the true space velocities may be different.. both in magnitude and direction. This implies that the selection has been performed in an erroneous way so that the distances which are derived from the spurious group motion may systematically be in error. If in reality the stars are not interrolated, a number of nearby stars $m$ zy have been included of which actually the velocity component in the direction of the supposed Apex is only small. At the same time a number of distant stars may have been included, having a large component of their space velocity in this same direction. If afterwards on the basis of the supposed common space velocity parallaxes are assigned to the individual stars, these parallaxes may be affected by systematic errors. For the nearby stars the distance will be overra. ted while that of the distant stars is underrated.

One of the probably spurious groups which has extensively been discussed is the well known Perseus group. It was first noted independently by Eddington [ $\left.{ }^{1}\right]$ and Boss [ $\left.{ }^{2}\right]$ who assigned 16 B type stars to the cluster. Later Rasmusson [ $\left.{ }^{3}\right]$ added a further list of 29 stars while more recently 2 further stars were added by Smart [4]. The latter author made an extensive and critical examination of the proper motions of the supposed group members. His conclusions are that the radial velocity criterion rules out a considerable proportion of the stars as members of a possible cluster, that his results are not definitely conclusive as to the real kinematical, characteristics of the investigated stars and finally that possibly a small nucleus of the stars may form a moving cluster. The parallaxes which he derives from the group motion and the resulting absolute magnitudes seem to be consistent with the commonly adopted values. When his parallaxes are compared with the spectroscopic ones it appears that the ratios $\pi$ spec : $\pi$ cal systematically depend on the distance of the stars considered. In section 2 this point will be considered in some detail. Obviously from the conclusions drawn by Smart it could be anticipated that such might be the case. The spectroscopic parallaxes may have been falsified by either the influ. ence of interstellar absorption or by an error in the zero point or eventually by a combination of the two effects. It may therefore be worth while to reconsider the motions of this group of 
stars, using a different -rethod in which the influence of $a b$ sorption is taken into account. This is the subject of the present paper.

\section{\$2. Comparison of the different parallaxes}

For applying the method to be used in the present paper it is essential only to use a group of stars of sufficient homogeneity, that is to say a group of stars with a relatively small range both in spectral type and in absolute magnitude. However, when comparing the spectroscopic parallaxes with the parallaxes which Smart has computed from the supposed group motion; no such restrictions need to be applied. Therefore, for all stars in the group for which values of both parallaxes are available, the spectroscopic parallaxes have been plotted against the corresponding calculated parallaxes.

The result appears in figure 1 . From the figure it is apparent that the individual points are not scattered along the

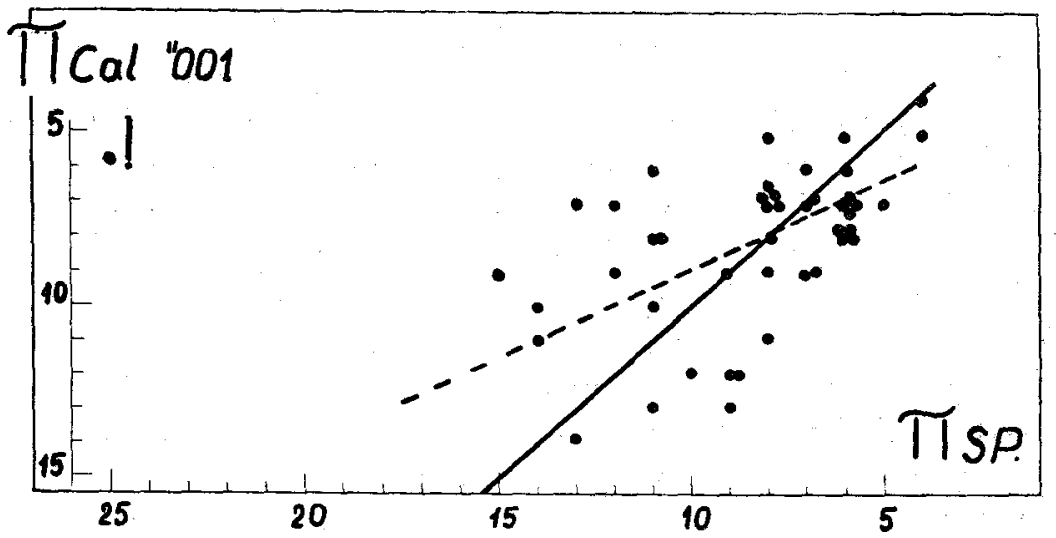

Fig. 1. Mean relation between the observed parallaxes and those calculated from group motion.

straight line $\pi_{s}=\pi_{c}$ which in the figure has been indicated by a full drawn line. Instead of this the points seem roughly to be scattered along the dotted line which has a different slope. In the numerical values of the ratios $\pi_{s} / \pi_{c}$ there is therefore a definite tendency to decrease with increasing distance. This may 
be taken directly to confirm the conclusions drawn by Smart, but this implies that his calculated parallaxes are affected by systematic errors $O_{n}$ the other hand, neither the spectroscopic parallaxes can be supposed to be free from systematic errors resulting from the effect of the interstellar absorption and a possible error in the determination of the zero point which may have resulted from this absorption. In the following it has been tried to find the corrections which on both accounts are to be applied to the spectroscopic parallaxes.

\section{\$ 3. Relations for determining the influence of interstellar absorption}

In order to have a homogeneous grpup of stars, in the following computations only the B type members of the Perseus group have been included. The method which is applied is a slight modification of one of those which have been considered in a recent paper [5]. It will be supposed that the relation between the true distance $r$ and the distance $y$ derived from the spectroscopic parallax is given by $r=y e^{a y}=y(1+a y)$ where the latter approximation is adequate for small distances. Obviously the coefficient $a=a(y)$ is closely related to the commonly used value $a(r)$. It is evident that $a$ can only assume negative values. If the necessary corrections have been applied to the observed values of $V_{R}, \mu_{x}$ and $\mu_{\delta}$ the relations between the components of the space velocity of the sun, that of the group velocity and the observed values are given by :

$$
\begin{aligned}
& -\mathrm{V}_{\mathrm{R} \odot}+\mathrm{V}_{\mathrm{R}_{p}}=\left\{\mathrm{V}_{\mathrm{R}}-y \cdot \mathrm{R}(l, b)-a y^{2} \cdot \mathrm{R}(l, b)\right\} \\
& -\mathrm{V}_{\alpha \odot} \odot \mathrm{V}_{\alpha p}=\left\{\mathrm{K}_{y} \mu_{\alpha}+\mathrm{K}^{2} a \mu_{x}\right\} \\
& -\mathrm{V}_{\delta \odot} \odot \mathrm{V}_{\delta p}=\left\{\mathrm{K}_{y} \mu_{o}+K_{y^{2} a \mu_{\delta}}\right\}
\end{aligned}
$$

Here $\mathrm{R}$ stands for the correction for differential galactic rotation $\mathrm{R}=\mathrm{A} \sin 2\left(l-l_{0}\right)$ and $\mathrm{K}=4.74$.

For the present no corrections for differential galactic rotation have been applied to $\mu_{\alpha}$ and $\mu_{\delta}$.

If the members of the Perseus group have a common space velocity, the terms which appear on the left hand side of the equations (1), (2) and (2) must be a constant. Therefore, these equations can be reduced to a much simpler form ; 
$\mathrm{X}_{\mathrm{I}}=\mathrm{V}_{\mathrm{R}}^{\prime}+a \mathrm{~A}_{1} ; \quad \mathrm{X}_{2}=\mathrm{V}_{x}^{\prime}+a \mathrm{~A}_{2}$ and $\mathrm{X}_{3}=\mathrm{V}_{\delta}^{\prime}+a \mathrm{~A}_{3} \cdots(4)$ and each star of the group will give a set of equations of the form (4).

$V_{R}^{\prime}, A_{1}, V_{x}^{\prime}, A_{2}, V_{\delta}^{\prime}$ and $A_{3}$ are known fron the observations and consequently $X_{1}, X_{2}, X_{3}$ and $a$ can be obtained from a leasi squares solution. Although the normal equations are well known and are not of great importance, they are still given here for future reference:

$$
\left.\begin{array}{rlrl}
{\left[\mathrm{V}_{\mathrm{R}}^{\prime}\right]} & =[1] \mathrm{X}_{1}-\left[\mathrm{A}_{1}\right] a & {\left[\mathrm{~V}_{\alpha}^{\prime}\right]=[1] \mathrm{X}_{2}-\left[\mathrm{A}_{2}\right] a} \\
{\left[\mathrm{~A}_{1} \mathrm{~V}_{\mathrm{R}}^{\prime}\right]} & =\left[\mathrm{A}_{1}\right]-\left[\mathrm{A}_{1}^{2}\right] a & {\left[\mathrm{~A}_{2} \mathrm{~V}_{\alpha}^{\prime}\right]=\left[\mathrm{A}_{2}\right] \mathrm{X}_{2}-\left[\mathrm{A}_{3}^{2}\right] a} \\
{\left[\mathrm{~V}_{\delta}^{\prime}\right]} & =[1] \mathrm{X}_{3}-\left[\mathrm{A}_{3}\right] a & & \\
{\left[\mathrm{~V}_{\delta}^{\prime} \mathrm{A}_{3}\right]} & =\left[\mathrm{A}_{3}\right] \mathrm{X}_{3}-\left[\mathrm{A}_{3}^{2}\right] a & &
\end{array}\right\}
$$

It should be noticed that $V_{x}$ is lineair in $y, A_{2}$ depends on the second power of $y, \mathrm{~A}_{2} \mathrm{~V}_{\alpha}^{\prime}$ on the third power of $y$ and $\mathrm{A}_{2}{ }^{2}$ on $g^{4}$.

When considering the equations $1-5$, it is evident that no good results can bo expected unless the terms $-V_{R} \odot+V_{R p}$; $-\mathrm{V}_{\alpha} \odot+\mathrm{V}_{p \chi}$ and $-\mathrm{V}_{\delta} \odot+\mathrm{V}_{\delta p}$ are really constant.

Due to the way the stars have been selected, this at least approximately may be the case with the tangential velocities. On the other hand from the conclusions given by Smart it may be inferred that with the radial velocities this condition may not be fulfilled. As is seen from the relation (1) the determina. tion of $a$ actually amounts to finding the variation of a differential term which in itself is already small. Therefore the expectancy of the residual term in $X_{1}$ is relatively so large that no value can be attached to the solution for $a$ obtained from the radial velocity, especially because the number of stars which is available is rather small. On the other hand any possible absorption will directly affect the computed values of the tangential velocities, so that it should be possible to detect this effect. 


\section{\$ 4. The determination of the numerical value of $a$.}

In table 1 all data needed for the solution have been collected. The number in the first column is the rotation number of the star in the list collected by Smart (1.c. table 3). The second column contains the distance $y$ as derived from the spectroscopic parallax and which therefore is based on the observed difference $M-m$ The third column contains the radial velocity $V_{R}^{\prime}$ This value $V_{R}^{\prime}$ is obtained from the observed value of the radial velocity by applying the constant correction for the $\mathrm{K}$ term, in this case $5 \mathrm{Km} / \mathrm{sec}$. and the correction for differential rotation $y R(l, b)$. Next the numerical values of $A_{1}$; $V_{R} ; A_{1} / 10$ and $\left(A_{1} / 10\right)^{2}$ are given. In the same way the necessary data for the tangential velocities have been entered in the successive columns of the table. It is to be observed that before computing the values $V_{\alpha}^{\prime}, A_{2}$ etc. to the observed values of the proper motions the corrections proposed by Wilson and Raymond ${ }^{6}{ }^{6}$ have been applied.

When the total values which are given at the bottom are inserted in the different equations, the following values are obtained:

$$
\begin{array}{ll}
\mathrm{X}_{1}=-3.8 \mathrm{~km} / \mathrm{sec} . & a=-0.00137 \quad \text { magn/parsec. } \\
\mathrm{X}_{2}=+9.2, \quad, \quad & a=-0.00069 \quad, \quad, \\
\mathrm{X}_{3}=-7.2, & a=-0.00083,
\end{array}
$$

If the weight of the individual solutions for $a$ is taken to be proportional to the numerical values of $A_{1}, A_{2}$ and $A_{3}$ the weighted mean value of $a$ appears to be

$$
a=-0.00077 \mathrm{magn} / \mathrm{parsec} \text {. }
$$

and using this latter value of $a$ the components of the space velocity are found to be :

$X_{1}=-3.2 \mathrm{Km} / \mathrm{sec} ; X_{2}=+8.1 \mathrm{Km} / \mathrm{sec} ; X_{3}=-8.0 \mathrm{Km} / \mathrm{sec}$.

The value obtained for $a$ is not impossible but seems to be too large. On the other hand the values which are obtained for the components of the space velocity are decidedly too small. This suggests that a correction may have to be applied to the zero point of the absolute magnitudes which have spectroscopically been determined. If this correction is $\Delta M, y$ the spec- 
troscopic distance before and $y^{\prime}$ the spectroscopic distance before and $y^{\prime}$ the spectroscopic distance after correction we would have $\lrcorner M=5 \log y / y^{\prime}$, so that the ratio $y^{\prime} / y$ is a constant.

\section{$\S 5$. Evalution of the ratio $y^{\prime} / y$}

The second set of the equations (5) could now be written :

$$
\begin{gathered}
{\left[\mathrm{V}_{\alpha}^{\prime}\right] y^{\prime} / y=[1] \mathrm{X}_{2}-\left[\mathrm{A}_{2}\right] a y^{\prime 3} / y^{3}} \\
{\left[\mathrm{~A}_{2} \mathrm{~V}_{\alpha}^{\prime}\right] y^{\prime} / y=\left[\mathrm{A}_{2}\right] \mathrm{X}_{2}-\left[\mathrm{A}_{2}{ }^{2}\right] a y^{\prime 3} / y^{2}}
\end{gathered}
$$

and when $X_{2}$ is eliminated between (6) and (7) the resulting equation is of the form :

$$
a=\mathrm{C}_{1} \times \frac{y^{2}}{y^{\prime 2}}
$$

and even a small correction to be applied to the scale of absolute magnitudes will have a considerable influence on the numerical value of $a$ which is obtained.

When next the value of $a$ as obtained from (8) is inserted in (6) it is evident that the value of $X_{2}$ is purely linear in $y^{\prime} / y$ Therefore if $X_{2}^{\prime}$ is the correct value of the velocity component, obtained by using the correct value of the distance $y^{\prime}$ we also have $\mathrm{X}_{2}^{\prime} / \mathrm{X}_{2}=y^{\prime} / y$. In the same way we find $\mathrm{X}_{3}^{\prime} / \mathrm{X}_{3}=y^{\prime} / y$.

Therefore if the ratios $X_{2} / X$ and $X_{3}{ }^{\prime} / X$ can be determined the value of $a$ can be further improved.

If we had to deal with a large number of stars chosen at random, the true values $X_{2}^{\prime}$ and $X_{3}^{\prime}$ would be equal to the components of the solar velocity in the directions of $\alpha$ and $\delta$. With the group of stars considered here the choice certainly has not been an at random one.

We have therefore adopted a space velocity of the group of $20 \mathrm{~km} / \mathrm{sec}$. in the direction of the apparent Apex at $\mathrm{A}=103^{\circ}$ and $D=-36^{\circ}$, the co ordinates given by Smart

The corresponding values of $\mathrm{X}_{2}^{\prime}$ and $\mathrm{X}_{3}{ }^{\prime}$ appear to be $\mathrm{X}_{2}{ }^{\prime}=+13.9$ and $\mathrm{X}_{3}{ }^{\prime}=-13.8$. The resulting values are $y^{\prime} / y=1.53$ and $y^{\prime} / y=1.92$.

For the present we will adopt the mean value $y^{\prime} \mid y=1.72$ Next for the corrected value of $a$ we find: 


$$
a=\left(\frac{1}{1.72}\right)^{2} \times 0.00077=-0.000266
$$

It is evident that the value (9) does not have the character of an accurate determination. For an accurate determination of both $y^{\prime} / y$ and $a$ the numbers of stars used here are altogether insulficient.

If we write :

$$
m=M+5 \log \frac{y^{\prime}}{10} e^{a g^{\prime}} M+5 \log \frac{y^{\prime}}{10}+a\left(y^{\prime}\right) y^{\prime}
$$

we readily find

$$
a\left(y^{\prime}\right)=-5 \times 0.000265 \log e=-0.00058
$$

In the usual relation

$$
m=M+5 \log \frac{r}{10}+a(r)
$$

this corresponds to

$$
a(r)=0.00103 \mathrm{magn} / \mathrm{parsec} \text {. }
$$

It is therefore apparent that the use of the spectroscopic parallaxes may lead to accurate estimates of the absorption, provided that sufficiently large numbers of stars are available to enable the determination of the numerical value $y^{\prime} \mid y$.

Judging from the present estimate $y^{\prime} / y=1.72$ the correction to be applied to the zero point of the scale of spectroscopically determined absolute magnitude should be $\Delta \mathbf{M}=-1 \cdot 2$. This correction is fairly large, but is not wholly unacceptable.

\section{\$ 6. Distances and absolute magnitudes}

Using the results of the preceding sections, the true distance of the stars is found to be

$r \doteq 1.72 y(1-0.00058 \times 1.72 y)=y^{\prime}\left(1-0.00058 y^{\prime}\right)$

while the absolute magnitude is equal to

$$
\mathrm{M}=m-5 \log \frac{y^{\prime}}{10}+0.00058 y^{\prime}
$$

In table 2 the resulting values of the parallax and of the absolute magnitudes are compared with those previously obtai- 
ned by other authors. In this table the stars are arranged in the same order as in table 1 . The columns 2,3 and 4 give the parallax computed from the equation (10), the spectroscopic parallax taken from the General Catalogue, and the parallax calculated by $\mathrm{Smart}(1, \mathrm{c})$ respectively. The next three columns give the absolute magnitude as obtained from the equation (11), the absolute magnitude as calculated by $S$ mart and the spectral type respectively. As is apparent from the table, the absolute magnitudes obtained by the present method are smaller than those obtained by Smart. That is to say, a higher luminosity is assigned to the different stars.

Only to a small extent can this difference be ascribed to the fact that in the paper by Smart the influence of absorption has been neglected. The main reason for this difference is that the present method leads to smaller values of parallax. Our impression is that on the whole our absolute magnitudes are slightly too bright.

The main weakness in the present paper is the uncertainty in the determination of the ratio $y^{\prime} \mid y=\mathrm{X}_{8}{ }^{\prime} / \mathrm{X}_{3}=\mathrm{X}_{2}{ }^{\prime} / \mathrm{X}_{2}$.

For $X_{3}$ ' and $X_{2}$ ' the "true, values of the components of the space velocities rather arbitrary selected values had to be used. If for the mean space velocity of the group a smaller value is adopted, the values of the absolute magnitudes are reduced, but such a smaller value of the space velocity cannot be reconciled with the results obtained by Smart.

For the present we can only conclude that according to the method used in this paper either the absolute magnitude of the stars in the Perseus group is higher than estimated by Smart or that the mean space velocity is smaller than the value found by him. 
Table II. Comparison of parallaxes and absolute magnitudes

\begin{tabular}{|c|c|c|c|c|c|c|}
\hline No. & $\begin{array}{c}\pi \\
(1.011)\end{array}$ & $\begin{array}{c}\pi_{s p} \\
(! .001)\end{array}$ & $\pi_{s m}$ & $\mathbf{M}$ & $M_{s m}$ & Type \\
\hline 15 & 3 & 4 & 4 & -1.8 & -0.5 & B 3 \\
\hline 25 & 3 & 4 & 5 & -2.5 & -1.2 & B 3 \\
\hline 2 & 4 & 5 & 8 & -22 & -0.2 & B 3 \\
\hline 48 & 4 & 5 & 7 & -1.6 & -00 & B 5 \\
\hline 3 & 4 & 6 & 7 & -2.0 & -0.8 & B 3 \\
\hline 6 & 4 & 6 & 7 & -1.5 & -0.0 & B $\mathbf{5}$ \\
\hline 11 & 4 & 6 & 6 & -1.8 & -0.6 & B 5 \\
\hline 12 & 4 & 0 & 7 & -4.1 & -2.8 & B 1 \\
\hline 14 & 4 & 6 & 8 & -2.2 & -0.5 & B 3 \\
\hline 16 & 4 & 6 & 8 & -2.0 & -0.5 & B $\mathbf{3}$ \\
\hline 37 & 4 & 6 & 7 & -1.5 & -0.1 & B 5 \\
\hline 36 & 4 & 6 & 6 & -16 & -0.6 & B 8 \\
\hline 38 & 4 & 6 & 6 & -1.5 & -0.6 & B 9 \\
\hline 1 & 4 & 7 & 9 & -1.5 & +0.1 & В 5 \\
\hline 4 & 4 & 7 & 6 & -1.6 & -0.8 & B 3 \\
\hline 5 & 5 & 8 & 7 & -1.7 & -0.8 & B 3 \\
\hline 7 & 5 & 8 & 7 & -1.9 & -1.0 & B 5 \\
\hline 9 & 5 & 8 & 7 & -2.3 & -1.5 & B 9 \\
\hline 8 & 5 & 8 & 8 & -0.0 & +0.6 & B 9 \\
\hline 18 & 5 & 8 & 7 & -3.6 & -0.7 & B 9 \\
\hline 21 & 6 & 9 & 9 & -0.8 & +0.3 & B 9 \\
\hline 23 & 6 & 9 & 12 & -0.9 & +0.8 & B 8 \\
\hline 26 & 7 & 11 & 8 & -25 & -2.0 & B 3 \\
\hline 31 & 7 & 11 & 8 & -0.5 & -0.2 & B 5 \\
\hline 17 & 7 & 11 & 10 & -1.0 & -0.1 & B 8 \\
\hline 13 & 8 & 13 & 7 & -1.5 & -1.6 & В $3 p$ \\
\hline 45 & 8 & 13 & 14 & -2.2 & -1.0 & B 3 \\
\hline 10 & 9 & 15 & 9 & -2.1 & -2.1 & B 5 \\
\hline
\end{tabular}




\section{Literature}

[1] Eddington, A.S.; M. N. 71, 43, 1910.

[2] Boss, B.; A. J. No. 620, 1910.

[3] Rasmusod, E.; Lund Medd. Ser. II, 26, 1921

[4] Smart, W. M.; M. N. 100, 560, 1940

[ ] Kreiken, E. A.; Comm. Ank. Astron. Inst. No. 21, 1957.

[6] Wilson and Raymond; Ap. J. 47, 57, 1938.

Astronomical Institute of Ankara University; October 1957

E. A. KREIKEN 